\title{
ASSESSMENT OF HIGHWAY BRIDGE UPGRADING BY DYNAMIC TESTING AND FINITE ELEMENT MODEL UPDATING
}

\author{
James Mark William Brownjohn ${ }^{1}$ \\ Pilate Moyo $^{2}$ \\ Piotr Omenzetter $^{3}$ \\ Yong $\underline{\mathrm{Lu}}^{4}$
}

\begin{abstract}
The Land Transport Authority of Singapore has a continuing program of highway bridge upgrading, to refurbish and strengthen bridges to allow for increasing vehicle traffic and increasing axle loads. One subject of this program has been a short span bridge taking a busy main road across a coastal inlet near a major port facility. Experiment-based structural assessments of the bridge were conducted before and after upgrading works including strengthening. Each assessment exercise comprised three separate components; a strain and acceleration monitoring exercise lasting approximately one month, a full-scale dynamic test carried out in a single day without closing the bridge and a finite element model updating exercise to identify structural parameters and mechanisms. This paper presents the dynamic testing and the modal analysis used to identify the vibration properties and the quantification of the effectiveness of the upgrading through the subsequent model updating. Before and after upgrade, similar sets of vibration modes were identified, resembling those of an orthotropic plate having relatively weak transverse bending stiffness. Conversion of bearings from nominal simple supports to nominal full fixity was shown via model updating to be the principal cause of natural frequency increases of up to $50 \%$. The utility of the combined experimental and analytical process in direct identification of structural properties has been proven and the procedure can be applied to other (e.g. larger) structures and their capacity assessments.
\end{abstract}

\footnotetext{
${ }^{1}$ Associate Professor and corresponding author

${ }^{2}$ Research Student

${ }^{3}$ Research Fellow

${ }^{4}$ Assistant Professor

Division of Structures and Mechanics, School of Civil and Structural

Engineering, Nanyang Technological University, 50 Nanyang Avenue, Singapore 639798, http://www.ntu.edu.sg/cse, tel: +65 67904773, fax: +65 67910676 email: cjames@ntu.edu.sg
} 


\section{PIONEER BRIDGE}

Pioneer Bridge (Figure 1a,b,c) was built along Pioneer Road in Western Singapore in 1968-70 and was designed for the loading of that era (Ministry of Transport, 1961) and on the basis of permissible stresses. The live loading specified in Ministry of Transport (1961) consists of a uniformly distributed load referred to as HA loading and an abnormal vehicle of gross weight $45 \mathrm{Mg}$, referred to as HB loading. The bridge span is $18.16 \mathrm{~m}$ between elastomeric bearings, which were designed as simple supports, and the width is $18.796 \mathrm{~m}$. The bridge crosses the outflow of a tidal storm drain into Jurong Port.

The bridge comprises 37 pre-cast pre-tensioned inverted T-beams, clearly visible in Figure 1b, tied together by 25 cast in-situ $203 \mathrm{~mm}$ thick transverse diaphragms at $762 \mathrm{~mm}$ centres. The T-beams carry a deck slab having thickness that varies from $152 \mathrm{~mm}$ to $305 \mathrm{~mm}$. Concrete cube strengths assumed in the original design were 42 MN.m $\mathrm{m}^{-2}$ for pre-tensioned girders and $26 \mathrm{MN} \cdot \mathrm{m}^{-2}$ for diaphragms and slab.

\section{BRIDGE ASSESSMENT AND STRENGTHENING WORKS}

As part of the Singapore Land Transport Authority (LTA) bridge management and upgrading program to cater for increased vehicle traffic and loading, the bridge was assessed to evaluate its strength and to identify any defects in the structure. British bridge assessment procedures laid out in documents BD 21/97, BA 55/94 and BD 44/95 (Highways Agency 1997, 1994, 1995) were adopted by LTA for the assessment of the load carrying capacity. BD 44/95 specifies use of 'worst credible strength' defined as the worst value of strength that can be obtained in the structural element under consideration, and for concrete elements this was estimated from LTA core tests yielding compressive strengths of $55 \mathrm{MN} \cdot \mathrm{m}^{-2}$ and $30 \mathrm{MN} \cdot \mathrm{m}^{-2}$ for T-beams and cast in-situ slab respectively.

The analytical assessment revealed that rubber bearings had been overstressed, but that the superstructure was capable of carrying vehicles of up to $44 \mathrm{Mg}$ gross mass as specified in BD21/97. However to maintain the load carrying capacity over the design life with loading specified by LTA, strengthening works were proposed in which the simply supported system would be converted to a jointless structure with the superstructure assumed to be continuous and monolithic with abutments. This would eliminate the need for bearings, increase redundancy and enhance load distribution at supports. In addition, this type of construction was found to suit site conditions where heavy vehicles had to continue to use the bridge during upgrading works in which a one-third width of the bridge at a time was closed and upgraded. 
Figure 1c shows the walkway of the bridge after upgrading. Visually the main differences after the upgrade are a tidier appearance and the addition of a massive guard rail between walkway, but a close inspection shows that the T-beams shown in Figure $1 \mathrm{~b}$ now appear to sit directly onto the abutment with no gap. In fact a series of heavy reinforcement bars now pass through the deck directly into the approach slab as shown in Figure 2 to provide rotational restraint.

It is interesting to note that while BD21/97 considers understanding of overall bridge structural behaviour important, (Part 3, BD21/97, Clause 3.2 \& Clause 3.29), according Clause 3.28 load testing is deemed unwarranted in most cases "because of the high costs involved, the possibility of causing structural damage while undertaking the tests and the difficulty in interpreting any test results".

This observation is especially surprising given the recognised value of full-scale static and dynamic testing (Bakht and Jaeger 1990). Developments in dynamic testing and signal processing are making it possible to gain economically considerable insight into structural behaviour without compromising structural integrity. The upgrading exercise provided the perfect opportunity to deploy and evaluate field testing equipment acquired for vibration measurements, and modal analysis software designed to work with both forced vibration and 'response only' vibration test data.

Dynamic testing of full-scale structures is, with some notable exceptions, rarely done by bridge owners/managers as it is still regarded as being too troublesome and expensive. Nevertheless there is a long history of its use on bridges (Carder, 1937) with more recent exercises providing practically useful information (e.g. Salawu \& Williams 1995, Samman \& Biswas 1994, Brownjohn \& Xia, 2000, Krishnan et al., 1996, Law et al., 1995) and some adoption by bridge managers. Along with increased activity there is a range of increasingly effective hardware and software tools and procedures available for modal testing and analysis. Coupled with the developing technology of finite element model updating (Cantieni 1996, Pavic et al. 1998, Brownjohn \& Xia 2000), there is a powerful set of tools for objective assessment of bridge structural condition.

There were thus three major objectives for the dynamic testing exercise. Firstly the aim was to demonstrate the capabilities of a combined testing and updating exercise. Secondly it was intended to use the experience as a prototype to learn and refine bridge assessment procedures. Third, some specific and quantitative feedback about the effectiveness of the upgrade was to be provided to LTA. 
The program comprised preliminary measurements to estimate the nature of the dynamic response followed by full-scale dynamic test to identify modal parameters before upgrading. The bridge was to be re-tested after upgrading, followed by finite element model updating to estimate pre- and post upgrade structural parameters.

\section{PRELIMINARY VIBRATION MEASUREMENTS}

Figure 3 shows time series of deck vertical accelerations induced by passing vehicles. From the part of the signal showing clear exponential decay after a vehicle has left the bridge, a decaying sinusoid was fitted, indicating a resonant frequency of $6.15 \mathrm{~Hz}$ and a damping ratio of $2.7 \%$. Another part of the data provided a frequency of 9.8Hz and damping of $4.1 \%$. Digital Fourier Transforms (FFTs) of longer time series showed other possible modes around $7 \mathrm{~Hz}, 10 \mathrm{~Hz}, 15 \mathrm{~Hz}$ and $21 \mathrm{~Hz}$. While the data contained strong elements of the forcing due to vehicle body bounce and axle hop, the observed frequencies turned out to correspond to valid modes.

\section{PRE-UPGRADE VIBRATION SURVEY, SUNDAY $8^{\text {TH }}$ OCTOBER 2000}

The preliminary measurements identified the ranges of frequency $\left(5-21 \mathrm{~Hz}\right.$ ) and amplitude (up to $0.5 \mathrm{~m} . \mathrm{sec}^{-2}$ ) for a more comprehensive vibration survey to obtain a definitive set of natural frequencies with associated damping ratios, mode shapes and modal masses. The complete operation of transporting equipment to site, deploying, testing, packing up and returning had to be done in a single day. Only one day could be found having low traffic (Sunday) and low tide for most of the day before the upgrading, which was scheduled to start on $9^{\text {th }}$ October.

A frequently cited objection to the use of vibration testing to assess structural condition is that such tests are either laboratory exercises allowing good control of environment and testing conditions, or are elaborate exercises on prototypes that have to be closed to normal use. In this case there was no interruption to the normal operation of the bridge and a prime objective of the test was to show what could be achieved under constrained circumstances.

A grid of points measuring only vertical acceleration was chosen, dividing the bridge into four strips in the transverse direction and six in the traffic direction. Locations along each edge were accessible via walkways on each (north and south) side of the bridge, the remainder were only accessible on the bridge soffit. The shaker was located at the one-third span position on one walkway for convenience and maximum effect.

The program intended to make a sequence of measurements with accelerometers arranged as follows:

Pi1: 13 locations along the north footpath

Pi2: 13 locations along the south footpath

Pi3: 7 locations along each footpath (total 14 accelerometers) 
Pi4_n: a sequence of five measurements $(n=1-5)$ each including locations in a transverse line across the bridge, sweeping from abutment to abutment.

In preliminary measurements (Pi1 and Pi2) using a full set of thirteen accelerometers along each walkway in turn, shaker excitation, vehicle-induced response (free vibration) and hammer testing were used to assess the viability of each procedure in providing quality data for system identification. As the best data were acquired when using a shaker, free vibration and hammer testing were discontinued.

Due to operational reasons mainly associated with time taken to pass instrument cables under the bridge, towards the end of the testing as a result of rain, wind, failing light and rising tide the complete sequence of measurements sweeping transducers under the bridge (pi_4) had to be curtailed. In the event, a convincing set of vibration frequencies and damping ratios were obtained, and making some assumptions about symmetry, enough data were available to construct a set of vibration mode shapes.

\section{VIBRATION MEASUREMENT PROCEDURE AND SUMMARY}

\section{Equipment}

Force balance accelerometers had been employed on numerous prototype tests (Brownjohn et al., 1992) and had always provided the best quality of signal. Sixteen units of QA-700 force balance accelerometers, having broadband resolution down to $10^{-5} \mathrm{~m} \cdot \mathrm{sec}^{-2}$ were used in both pre- and post-upgrade testing. Due to operational limitations on the QA-700s, a set of Endevco 7754-1000 piezo-electric accelerometers was used for measurements on the bridge soffit. An APS 400 long stroke electro-dynamic shaker provided the broad-band excitation.

\section{Ambient vibration testing}

In recent years a number of procedures (Juang \& Pappa, 1985, James et al., 1995) have become available to civil engineers for identifying mode shapes, natural frequencies and damping ratios from 'response only' measurements. In such cases the response is to excitation that is unknown but whose force spectrum is usually assumed to be uniform (flat) or slowly varying with respect to frequency. For the type of excitation observed during the testing, the forcing was highly dependent on the dynamics of the vehicle(s) crossing the bridge with frequencies of structural response in resonance shifted through participation of heavy vehicles. 
It was however discovered that by examining short transients of response induced by light vehicles using the natural excitation technique (James et al., 1995), reasonable estimates of mode shape and frequency, if not damping, could be obtained.

\section{Shaker testing}

The best quality data were obtained from forced vibration testing with the shaker, which is designed to provide dynamic forces up to $0.5 \mathrm{kN}$ at low frequencies, between $1.5 \mathrm{~Hz}$ and $30 \mathrm{~Hz}$, which is exactly the range of concern in Pioneer Bridge. The shaker is shown in use (but in the post-upgrade testing) in Figure 1c.

Some trial and error was used to establish the optimum operation with a repeating chirp signal sweeping from $5 \mathrm{~Hz}$ to $32 \mathrm{~Hz}$ in a frame lasting 20.48 seconds with sample rate of $100 \mathrm{~Hz}$, and with ramp at start and end to avoid transients. The lower limit of $5 \mathrm{~Hz}$ was just below the $5.4 \mathrm{~Hz}$ bridge fundamental frequency.

As the bridge was not closed, the test was conducted during a period when the traffic was relatively low. Even so, less than $30 \%$ of recorded frames featured clean response due solely to the shaker. Other frames were affected by passing vehicles, and these were rejected during the averaging and smoothing procedure (Welch, 1967) the result of which was, for each measurement of $n$ channels, a $n \times n$ matrix of cross-power spectra (CPS). As a standard result, the frequency response function (FRF) between excitation and response is obtained by dividing CPS values by the auto-power spectrum of the input signal.

\section{Frequency response functions and modal mass}

Figure 4 shows the real and imaginary parts and the Nyquist plot of the frequency response function (FRF) between location 5 (accelerometer) and location 60 (shaker). The form of FRF that relates acceleration $\ddot{X}_{j}(\omega)$ at point $j$ to force input $P_{k}(\omega)$ at point $k$ at each frequency $\omega$ is defined as inertance and is defined, by summing contributions from all $N$ modes, as

$$
H_{j k}(\omega)=\ddot{X}_{j}(\omega) / P_{k}(\omega)=\sum_{r=1}^{N} \frac{-\omega^{2 r} \phi_{j}{ }^{r} \phi_{k}}{-\omega^{2}+\omega_{r}^{2}+2 i \zeta_{r} \omega_{r} \omega}, \quad \text { Equation } 1
$$

where ${ }^{r} \phi, \omega_{r}, \zeta_{r}$ are mode shape, natural frequency and damping ratio for mode $r$.

The component of FRF at mode $r$ comprises a dynamic amplification factor 


$$
H_{r}(\omega)=\frac{-\omega^{2}}{-\omega^{2}+\omega_{r}^{2}+2 i \zeta_{r} \omega_{r} \omega}
$$

having a maximum amplitude $0.5 / \zeta_{r}$ at resonance and a modal constant ${ }^{r} A_{j k}$ defined via equation 1 as

$$
{ }^{r} A_{j k}={ }^{r} \phi_{j}^{r} \phi_{k}
$$

Equation 3

for mass-normalised mode shapes and

$$
\begin{array}{ll}
{ }^{r} A_{j k}={ }^{r} \psi_{j}{ }^{r} \psi_{k} /{ }^{r} \psi \mathbf{M}^{r} \psi & \text { Equation } 4
\end{array}
$$

for measured mode shapes that are usually normalised to a maximum value of unity.

The relationship between measured and mass-normalised mode shapes is thus

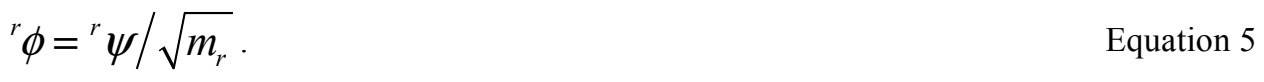

From equations 4,5 we obtain a definition of 'modal mass' $m_{r}$ whose value is computed using a mass distribution that can, for example, be represented via a discretised mass matrix $\mathbf{M}$ :

$$
m_{r}={ }^{r} \psi^{T} \mathbf{M}^{r} \psi, \quad \text { Equation } 6
$$

The definition of modal mass given in equation 6 depends on the mode shape scaling and can be used as in equation 5 to fix the scaling.

The SDOF circle fit procedure (Maia et al, 1997) was applied to the inertance of Figure 4 in distinct frequency bands, providing a very clear set of modes. Frequency and damping ratio estimates for a set of modes from 5-30 $\mathrm{Hz}$ are given in columns 4 and 6 of Table 1 and were obtained from the complete set of FRFs including Figure 4.

The circle fit to the Nyquist plot of Figure 4 around mode 3 (at 7.44Hz), provides a modal constant ${ }^{3} A_{5,60}=$ $2.5 \times 10^{-5} \mathrm{~kg}^{-1}$. This means that when forcing vibration in mode 3 at location 60 and measuring at location 5 , the bridge appeared to have a mass of $400 \mathrm{Mg}$. Location 5 was adjacent to the shaker so Figure 4 and the derived modal constant are effectively 'driving point inertance'.

The modal mass for the bridge corresponding to unit mode shape at the shaker is therefore $400 \mathrm{Mg}$ i.e. 400 metric tons. Given such values for modal mass, the linear behaviour of the bridge can be modelled as a set of 
SDOF oscillators with the possibility to predict bridge response to dynamic loads at any frequency and at any point via the mode shapes.

\section{RECOVERY OF MODE SHAPES}

While frequencies and damping values in themselves are useful and can be obtained from 'ad hoc' measurements such as the preliminary measurements reported earlier, it is worthwhile taking the considerable extra trouble to map out the mode shapes over a grid of points. Having identified the structural vibration modes, the modal masses and mode shapes can be used in response calculations or in model updating.

A further use for mode shapes that is relevant to condition assessment is their ability to show, through study of slope and curvature, the effectiveness of fixity at supports and the stiffness distribution, which can in extreme cases indicate defects or deficiencies.

To obtain mode shapes requires a comprehensive set of measurements all over the entire structure. In some cases (Farrar et al., 2000) it may be possible to install a large number of accelerometers to measure response of the bridge simultaneously, but for practical reasons most researchers use a smaller set of accelerometers (often limited to 16). The principle of vibration testing using relatively few sensors has been applied for several decades and involves maintaining the input force (for forced vibration testing) or reference accelerometer (for ambient vibration testing) at the same location while 'roving' other accelerometers between measurements to cover all possible measurement locations. The pieces of mode shape obtained from each measurement can be 'glued' (Hermans and Van der Auweraer, 1999) to provide the full mode shape at each frequency.

In the operation of gluing, the frequency response or transfer functions with respect to a common reference are merged before recovery of the amplitudes and phases from a single set of frequency response functions or normalised cross-powers.

\section{SYSTEM IDENTIFICATION USING ERA}

The eigensystem realisation algorithm or ERA (Juang \& Pappa, 1985) is a procedure for extracting a complete set of modal parameters from the cross-correlation functions between measured vibration signals which represent the impulse response functions of the structure. The impulse response functions can be obtained from time domain i.e. directly from the cross-correlation functions, or from the inverse FFT of either CPS or FRF data.

For Pioneer Bridge, as the force spectrum was flat, ERA was applied to the CPS involving only the response channels. For each measurement of 16 channels a CPS matrix was obtained using the Welch method with zero overlap, no windowing ands using only those valid frames containing no traffic excitation were used. As the only 
common reference signal between all the measurements was the shaker signal, columns of cross-powers with respect to the shaker were extracted from each CPS matrix, normalised by dividing by the shaker auto-power and then combined into a single column.

The resulting set of combined FRFs was converted back to CPS by multiplying the merged column of FRFs by the averaged reference auto-power spectrum. Data from four measurements: pi3, pi4_1, pi4_2 and pi4_3 were combined using mapping files and ERA was carried out using 150 data points of the impulse response function sampled at $100 \mathrm{~Hz}$, to yield the frequency and damping estimates given in columns 5 and 7 on Table 1 . Numerical quality indicators are high for these modes and they agree well with those from the circle fitting.

The modes recovered by ERA are classified in Table 1 columns 2 and 3 according to the number of vibration nodes in $\mathrm{x}$ (traffic) and y (transverse) directions. Figure 5 shows five modes so recovered at frequencies below $20 \mathrm{~Hz}$. These have half-sine pattern in the traffic direction with increasing order in the transverse direction. The black dots show where measurements were made and the hollow dots show where modal ordinates were not directly measured but were 'borrowed' from symmetric positions. Many locations on the west side of the bridge were not measured due to lack of time, but the nature of the modes and their symmetry is still evident from the successful measurements. Figure 6 shows higher order modes above $20 \mathrm{~Hz}$ using only points along the walkways; patterns in the transverse direction are too complex to distinguish due to the limited measurement points. Figure 4 shows that the modes are becoming broad and relatively indistinct above $10 \mathrm{~Hz}$.

\section{CONCLUSION FROM PRE-UPGRADE VIBRATION TEST}

The different forms of testing and analysis provided the same information: there was a set of five vibration modes in the range $0-20 \mathrm{~Hz}$ with half-sine shape in traffic direction, with a weakly excited fundamental at $5.4 \mathrm{~Hz}$. The modes showed a clear pattern of increasing complexity in the transverse direction, consistent with relatively lower stiffness in this direction. Above $20 \mathrm{~Hz}$ modes had a full-sine pattern in the longitudinal direction. For the abutments, the mode shapes had non-zero slopes showing the lack of restraint.

\section{POST-UPGRADE VIBRATION SURVEY, $18^{\mathrm{TH}}$ MARCH 2001}

Upgrading works commenced the day after the pre-upgrade testing, and the exercise of $8^{\text {th }}$ October 2000 was repeated as soon as possible after completion of these works. As for the earlier test, all the testing was done on a single day (again, a Sunday). Based on the lessons learnt from the pre-upgrade test, only shaker testing was conducted, while a greater resolution of measurement points between walkways i.e. under the bridge was provided to resolve the spatial complexity of the modes. Extra spatial resolution of measurement points at the 
abutments was also provided to allow for identification of the mode shape curvature at the supports which would indicate the effectiveness of the added fixity.

Figure 7 shows a driving point FRF i.e. using an accelerometer very close to the shaker. With respect to the preupgrade measurements, the lower mode frequencies appear to be significantly higher and the peaks broader, and in the range $20-30 \mathrm{~Hz}$ the response is dominated by one very broad mode at approximately $28 \mathrm{~Hz}$. The same procedure of frequency and damping estimation by circle fitting then verification with mode shape recovery using ERA was applied and a set of modes identified as shown in Table 2.

Figure 8 shows the set of corresponding mode shapes for the six clearest modes and Figure 9 shows the elevation view along the walkways. The partial fixity at the supports is evident. An immediate observation is that the same families of shapes appear, with those below $20 \mathrm{~Hz}$ once again having an approximate half-sine mode shape in traffic direction.

\section{COMPARISON WITH PRE-UPGRADE MODES}

Numerical comparison is made between pre-upgrade modes and post-upgrade modes in Table 2 columns 8-10. Modal assurance criterion or MAC (column 9) is numerically evaluated as a correlation coefficient between modal ordinates of a pair of modes. Well correlated modes generally have MAC $>0.9$, poorly correlated modes have $\mathrm{MAC}<0.5$.

For a given post-upgrade mode, the closest match with any pre-upgrade mode is indicated by the highest MAC. For modes 1-5 it is easy to find a convincing match and to compute percentage increases in frequencies (column 10). For modes above $20 \mathrm{~Hz}$ the correspondence is less clear. It also appears the corresponding modes have higher damping ratios after the upgrade. For both pre- and post-upgrade testing, the damping identified using ERA for modes above $20 \mathrm{~Hz}$ are mostly rather low and inconsistent with circle fit values which are likely to be more reliable.

The modal constant for the driving point inertance in mode $3(10.71 \mathrm{~Hz})$ is $2.5 \times 10^{-6} \mathrm{~kg}^{-1}$ for an effective mass of $400 \mathrm{Mg}$, identical to the pre-upgrade value. This can be compared with the bridge total mass estimated at 680 $\mathrm{Mg}$. 


\section{STRUCTURAL ASSESSMENT BY FINITE ELEMENT MODEL UPDATING}

\section{Theory}

The assessment described here is based on systematic comparison of the full-scale dynamic test data with dynamic characteristics obtained via finite element modeling (FEM). While FEM is an efficient analytical tool, creating a model capable to reproduce the measured dynamic characteristics of the prototype is a challenge. Discrepancies between experimental and analytical dynamic characteristics arise due to assumptions made in modeling, as well as uncertainties in material and geometrical properties and boundary conditions. Given that experimental results represent the real structure, FE model updating aims at improving systematically the structural model based on experimental results.

Model updating begins with selection of experimental responses such as natural frequencies and mode shapes to be used as reference data. The next step is the creation of the initial FE model, in which care must be taken to provide for grouping of uncertain structural parameters in a form accessible for updating. It seems a truism that the parameters for updating must be uncertain in the model, for example Young's modulus for concrete is uncertain while for steel it would be nonsense to look for a small change in a well established value. From the mathematical point of view, the number of parameters should be kept small to avoid ill-conditioning. Also, those parameters should be selected to which the responses are sensitive, thus guaranteeing a genuine improvement in the analytical model. The final step of the updating procedure is the model tuning, i.e. minimizing the discrepancy between analytical and experimental responses by successively adjusting the parameter values. In this study, model tuning is based on the sensitivity matrix.

Denoting the vectors of experimental and analytical responses as $R_{e}$ and $R_{a}$, respectively, one can express changes in the analytical responses due to changes in the parameters using the Taylor expansion limited to the linear term as:

$R_{e}=R_{a}+S\left(P_{u}-P_{0}\right)$ Equation 7

Vectors $P_{0}$ and $P_{u}$ denote updated and current parameter values, respectively, whereas $S$ is the sensitivity matrix defined as:

$$
S_{i j}=\frac{\partial R_{a, i}}{\partial P_{j}}
$$

Equation 8 
$S$ is typically rectangular and the updated parameters that yield the minimum least squares residue of the response error are usually found. As defined in Equation 8, S reflects absolute sensitivities of the responses to absolute parameter changes, and to observe relative changes in responses due to relative changes in parameters, a normalised relative sensitivity, $S_{n r}$, is used:

$$
\begin{array}{ll}
S_{n r}=R_{D, a}^{-1} S P_{D} & \text { Equation } 9
\end{array}
$$

where $R_{D, a}$ and $P_{D}$ are square, diagonal matrices holding response and parameter values, respectively. The normalized relative sensitivities are convenient for selecting the "active" parameters for updating.

\section{Numerical results and discussion: Pre-upgrade updating}

The updating exercise was conducted for the bridge before and after upgrading. The bridge before upgrading was modeled using 3D beam elements, assuming that T-beams are simply supported on the abutments. The structural mass was assessed using the as-built bridge drawings. The values of the Young's modulus for the structural elements were assessed using the worst credible strengths used by LTA in the load capacity assessment (Highways Agency 1995), giving respectively 34.9 GN.m ${ }^{-2}$ for the T-beams and 28.1 GN.m ${ }^{-2}$ for the slab and diaphragms.

Preliminary simulations showed that dynamics of the bridge were mainly governed by vertical bending stiffness of the structural members. Assuming a perfect bond between the T-beams and the slab, the vertical bending stiffness of such a compound beam was calculated as $E I_{T}=1.06 \times 10^{9} \mathrm{~N} \cdot \mathrm{m}^{2}$. Regarding the diaphragms, their bottom reinforcement consists of only one bar of diameter of $22 \mathrm{~mm}$, and hence the bending stiffness was calculated under the assumption that concrete does not carry any tension and the diaphragm cross-section is fully cracked, yielding the value of $E I_{D}=4.19 \times 10^{7} \mathrm{~N} . \mathrm{m}^{2}$.

The responses selected were the ratios $f_{a} / f_{e}$ of the first five analytical natural frequencies $f_{a}$ to their experimental counterparts $f_{e}$. In addition to these responses, MAC values were computed as useful indicators of correlation between experimental and analytical results. The parameters chosen for updating were stiffness of the T-beams and diaphragms with initial normalized relative sensitivities given in Figure 10. As could be expected from the mode shapes (see Table 1 and Figure 4), the lower mode frequencies depended mainly on T-beam stiffness and higher mode frequencies depended increasingly on diaphragm stiffness. The results of updating are summarized in Table 3, with pairing of experimental and analytical frequencies shown in Figure 11. Significant improvement in agreement between the first five analytical frequencies and their experimental counterparts can be seen after 
updating, the largest discrepancy being about 5\%. On the other hand, the MAC values remain almost unchanged and relatively low. For the higher frequency modes, updating is not particularly successful; e.g. it predicts a mode not identified by the test, and for these modes that have been identified by the test the discrepancies in frequencies and modal shapes are generally higher.

After updating the final parameters were $E I_{T}=1.32 \times 10^{9} \mathrm{~N} . \mathrm{m}^{2}$ and $E I_{D}=6.87 \times 10^{7} \mathrm{~N} . \mathrm{m}^{2}$, increases of $24.5 \%$ and $64.0 \%$ respectively as compared to the initial values and still in an acceptable range. From the point of view of structural assessment of the bridge it is important to recognize that diaphragms have relatively low stiffness and therefore it can be expected that redistribution of live loads is poor.

\section{Post-upgrade updating}

The stiffness of the T-beams and diaphragms for the upgraded bridge were taken as values obtained through updating of the pre-upgrade model. To reflect the structural change in the upgrading, the abutment restraints were modeled as rotational springs of finite stiffness. Preliminary simulations showed that a reasonable starting value for rotational stiffness should be of order $k_{R}=10^{8} \mathrm{~N} \cdot \mathrm{m} \cdot \mathrm{rad}^{-1}$. Another major structural enhancement due to upgrading was the provision of a concrete vehicular impact guard rail (Figure 1c). Initial simulations suggested that the guard rail should be modeled as an independent beam with no connection able to transmit shear forces from the slab, with vertical bending stiffness computed as $E I_{G}=6.02 \times 10^{8} \mathrm{~N} . \mathrm{m}^{2}$.

The responses selected were once again the ratios of the first five analytical natural frequencies to their experimental counterparts. Initially updating of two parameters, i.e. stiffness of guard rail and restraining springs was attempted. The normalized relative sensitivities are shown in Figure 12 and it can be seen that the sensitivity for spring stiffness is much greater than for guard rail stiffness. The two-parameter updating considerably improved the frequency values but at the same time reduced the MAC values and identified unreasonably high guard rail stiffness. Hence the guard rail stiffness was fixed leaving the only "active" parameter to be spring stiffness, which was updated to $k_{R}=4.15 \times 10^{8} \mathrm{~N} \cdot \mathrm{m} \cdot \mathrm{rad}^{-1}$. Further results of updating are shown in Table 4. Pairing of experimental and analytical frequencies is also shown in Figure 13. This shows improved correlation with frequency errors generally less than $10 \%$ although the MAC values remain almost unchanged and relatively low. The experience suggests that a more effective updating strategy should include MAC or COMAC (coordinate MAC) values in the response vector. For higher frequency modes, FEM was not particularly successful in prediction of experimental data. 
These investigations showed that bridge stiffness increased considerably due to the upgrading. This is evident from the increase of first natural frequency from $5.55 \mathrm{~Hz}$ to $8.31 \mathrm{~Hz}$. Additional simulation with fully fixed ends of the T-beams yielded a fundamental natural frequency of $12.21 \mathrm{~Hz}$ leading to the conclusion that although the bridge has not been fully restrained against rotations at the abutments, partial fixity has been attained. The partial fixity after upgrading is also evident in Figure 14 which compares the first experimental and analytical mode shapes along one of the span edges.

\section{DISCUSSION}

The purpose of the exercise has been to show how dynamic testing procedures coupled with finite element modelling and systematic updating could be used to provide quantitative evidence showing the effect of the upgrading works. Beyond that, a FE model of the bridge before and after the upgrading is now available that can be used to estimate load carrying capacity more reliably than would a pure desk exercise which has to make a number of simplifications or assumptions based on drawings or visual inspection. The procedure has now been proven and we hope to use it to assist LTA in their bridge assessment and upgrade program. The remaining question is whether such assistance can be cost-effective, which is why some constraints on timing and complexity of analysis have been applied.

We chose to use dynamic testing and dynamics-based model updating because the testing procedure is generally simpler, cheaper and less intrusive than static testing. Also the tools for model updating based on dynamic properties are relatively well developed. We do not rule out the use of static or quasi-static response data to assist in the assessment, in fact we have in parallel used a temporary strain monitoring system to assess the statistical distribution of live strains before and after the upgrading (Moyo et al., 2002).

Dynamic testing procedures for civil structures have evolved rapidly in recent years due largely to improved system identification procedures. The testing and data analysis used here make use of and adapt these newer technologies. For the size of bridge tested it is clear that forced vibration testing using portable and commercially available shakers was the best procedure. For larger bridges this would not be the case as the response that could be generated by these shakers would be too small by comparison with background noise including ambient excitation by wind or vehicles. In such cases, without the luxury of purpose-built shakers and the expensive logistical exercise required to transport and operate them, and with the likely need to close the bridge during the testing, ambient vibration testing is the best bet. Happily, for larger bridges, the dynamic interaction between the 
structure and the vehicles reduces and it becomes simpler and more practical to use ambient or 'output only' testing, a procedure that is now very well developed.

\section{CONCLUSIONS}

The experimental program clearly demonstrated the viability of non-invasive full-scale dynamic testing allied with finite element model updating for assessing modal and structural parameters of a highway bridge.

The modal characteristics, specifically the set of modes with half-sine shape in the traffic direction, clearly demonstrated the orthotropic character of the deck. During tests before and after upgrading of the bridge it was harder to identify modes for higher order (full-sine) vibration modes, but an adequate description of modes up to at least $20 \mathrm{~Hz}$ was obtained, and modal properties obtained using two identification techniques were consistent.

The effects of two major structural changes i.e. rotational restraint at the abutment and large guard rail were clear from the significantly increased natural frequencies. A model updating exercise used the experimental data to identify appropriate values of girder stiffness in the two directions and to show that the guard rail was a smaller influence on the post-upgrade performance.

The application of the procedure to a relatively simple bridge showed clearly the potential for such a procedure to assist bridge managers assess their structures by providing validated structural models that may be used for load capacity assessment. For larger bridges it may be more appropriate and simpler to use ambient or 'output only' testing, but the same identification and updating processes could be applied.

\section{ACKNOWLEDGEMENTS}

The authors would like to thank Tan Boon Leong, Lau Hwa Cheong and Chuai Chip Tiong of LTA, and Mr Jayaraman of PBMM for providing structural information and the opportunity for the research. Many thanks to Dr Tan, Poh and Max of SysEng Ltd, Xia Pinqi, Giovanna Zanardo, Desmond Ong and Ng Wee Boon, without whose help the testing would have been impossible.

\section{REFERENCES}

Bakht B., Jaeger L. G., (1990). “Bridge testing -a surprise every time.” Journal of Structural Engineering, ASCE, 116(5), 1370-1383.

Brownjohn, J. M. W. and Xia, P. Q. (2000). "Dynamic assessment of curved cable-stayed bridge by model updating.” Journal of Structural Engineering, ASCE, 126(2), 252-260. 
Brownjohn, J. M. W., Dumanoglu, A. A., and Severn, R. T. (1992). “Ambient vibration survey of the Fatih Sultan Mehmet (Second Bosporus) suspension bridge." Earthquake Engineering and Structural Dynamics, John Wiley \& Sons, Ltd., 21, 907-924.

Cantieni, R., (1996). "Updating of analytical models of existing large structures based on modal testing.” Proc., Workshop US-Europe on Bridge Engrg.: Evaluation Mgmt. and Repair, ASCE, Reston, Va., 15-17.

Carder, D. S., (1937) Observed vibrations of bridges. Bulletin, Seismological Society of America, 27 no. 4 , 267-303.

Doebling, S. W., Farrar, C. R., and Prime M. B. (1998) “A summary review of vibration-based damage identification methods.” The Shock and Vibration Digest, 30(2), 9-105.

Farrar, C.R., Cornwell, P.J., Doebling, S.W., Prime, M.B., (2000). Structural Health Monitoring Studies of the Alamosa Canyon and I-40 Bridges, Los Alamos National Laboratory Report LA-13635-MS.

Hermans, L. and Van der Auweraer, H., (1999). "Modal testing and analysis of structures under operational conditions: industrial applications" Mechanical systems and signal processing 13(2), 193-216.

James, G. H., Carne, T. G., Lauffer, J. P., (1995). “The natural excitation technique (NExT) for modal parameter extraction from operating structures", Journal of Analytical and Experimental Modal Analysis 10(2), 260-277. Juang, J-N., Pappa, R. S., (1985). “An eigensystem realisation algorithm for modal parameter identification and model reduction", Journal of Guidance (AIAA) 8(5), 620-627.

Krishnan, K., Seible, F. and Pardoen, G., (1996). "Evaluation of bridge strengthening measures using forced vibration tests.” ASCE Structures Congress, V2, 845-849

Law, S. S., Ward, H. S., Shi, G. B., Chen, R. Z., Waldron, P. and Taylor, C.A. (1995) “Dynamic assessment of bridge load-carrying capacities. 1 \& 2”. Journal of Structural Engineering, ASCE, 121(3), 478-495.

Maia, N. M. M. , Silva, J. M. M. , He, J., Lieven, N. A. J., Lin, R. M., Skingle, G. W., To, W. and Urgueira, A. P. V. (1997) . “Theoretical and Experimental Modal Analysis.” Research Studies Press Ltd.

Moyo P., Brownjohn J. M. W., Omenzetter P., (2002) "Highway bridge live loading assessment and load carrying capacity assessment using a health monitoring system." Proceedings, First European Conference on Structural Health Monitoring, Paris. 
Pavic, A., Hartley, M. J., and Waldron, P. (1998). "Updating of the analytical models of two footbridges based on modal resting of full scale structures.” Proc., 23rd Int. Seminar on Modal Analysis, Society for Experimental Mechanics, Inc., Bethel, 1111-1118.

Salawo, O. S., Williams C., 1995, "Bridge assessment using forced vibration testing" Journal of Structural Engineering, ASCE, 121(2), 161-172.

Samman, M. M., Biswas. M., (1994). "Vibration testing for nondestructive evaluation of bridges 1: testing \& 2: results", Journal of Structural Engineering, ASCE, 120(1), 269-306.

The Highways Agency (1994). Design manual for roads and bridges Vol. 3 Highway Structures: Inspection and maintenance Section 4 Assessment Part 9 BA 55/94. The assessment of bridge substructures and foundations, retaining wall and buried structures. HMSO, London, UK

The Highways Agency (1995). Design manual for roads and bridges Vol. 3 Highway Structures: Inspection and maintenance Section 4 Assessment Part 14 BA44/95. The assessment of concrete highway bridges and structures. HMSO, London, UK

The Highways Agency (1997). Design manual for roads and bridges Vol. 3 Highway Structures: Inspection and maintenance Section 4 Assessment Part 3 BD 21/97. The assessment of highway bridges and other structures.

HMSO, London, UK

United Kingdom Ministry of Transport (1961). Standard Highway Loadings. Memo No. 771, HMSO, London, UK.

Welch, P.D., (1967). "The use of fast Fourier transform for the estimation of power spectra: A method based on time averaging over short, modified periodograms.” IEEE Transactions, AU-15, 70-73 
Table 1 Pre-upgrade experimental modal parameter estimates from circle fitting and eigensystem realisation algorithm (ERA)

\begin{tabular}{|l|l|l|l|l|l|l|}
\hline \multirow{2}{*}{ Mode } & \multicolumn{2}{|l|}{ Mode type } & \multicolumn{2}{l|}{$\mathrm{f}_{\mathrm{e}} / \mathrm{Hz}$} & $\zeta / \%$ \\
\cline { 2 - 7 } & $\begin{array}{l}\text { Nodes } \\
\text { in } \mathrm{x}\end{array}$ & $\begin{array}{l}\text { Nodes } \\
\text { in y }\end{array}$ & Circle fit & ERA & Circle fit & ERA \\
\hline 1 & 0 & 0 & 5.47 & 5.55 & 3.5 & 2.6 \\
\hline 2 & 0 & 1 & 6.27 & 6.26 & 2.3 & 1.8 \\
\hline $3 \mathrm{a}$ & 0 & 2 & - & 7.31 & - & 3.9 \\
\hline $3 \mathrm{~b}$ & & & 7.44 & 7.53 & 2.0 & 1.8 \\
\hline $4 \mathrm{a}$ & 0 & 3 & & 10.13 & & 2.2 \\
\hline $4 \mathrm{~b}$ & & & 10.32 & 10.30 & 3.2 & 2.8 \\
\hline 5 & 0 & 4 & 15.02 & 14.76 & 3.0 & 2.5 \\
\hline 6 & 1 & - & 20.10 & 20.81 & 3.6 & 0.7 \\
\hline 7 & 1 & 4 & 23.23 & 23.74 & 4.0 & 0.5 \\
\hline 8 & 1 & - & 29.33 & 30.74 & 2.1 & 0.95 \\
\hline
\end{tabular}

$f_{e}=$ experimental frequency; $\zeta=$ damping ratio 
Table 2 Post-upgrade experimental modal parameter estimates from circle fitting and eigensystem realisation algorithm (ERA) and comparison with pre-upgrade modes.

\begin{tabular}{|l|l|l|l|l|l|l|l|l|l|l|}
\hline & \multicolumn{3}{l}{ Mode type } & \multicolumn{2}{l|}{$\mathrm{f}_{\mathrm{e}} / \mathrm{Hz}$} & \multicolumn{2}{l|}{$\zeta \%$} & \multicolumn{2}{l|}{ Post-pre comparison } \\
\hline$\#$ & Nodes in $\mathrm{x}$ & Nodes in y & Circle fit & ERA & Circle fit & ERA & Pre-mode \# & MAC & $\Delta f \%$ (ERA) \\
\hline 1 & 0 & 0 & - & 8.31 & - & 5.5 & 1 & 0.96 & +49.7 \\
\hline 2 & 0 & 1 & 9.27 & 9.34 & 3.8 & 4.2 & 2 & 0.89 & +49.2 \\
\hline 3 & 0 & 2 & 10.71 & 10.71 & 1.8 & 1.9 & $3 \mathrm{a}$ & 0.94 & +46.5 \\
\hline 4 & 0 & 3 & 12.82 & 12.95 & 2.0 & 2.0 & $4 \mathrm{a}$ & 0.81 & +27.8 \\
\hline 5 & 0 & 4 & 17.10 & 17.15 & 3.0 & 2.5 & 5 & 0.94 & +16.1 \\
\hline 6 & 1 & - & - & 21.02 & - & 0.8 & & & \\
\hline 7 & 1 & - & - & 26.89 & - & 0.6 & & & \\
\hline 8 & 1 & - & 28.22 & 27.91 & 5.0 & 4.8 & 6 & 0.64 & +23.1 \\
\hline 9 & - & - & - & 32.91 & - & 1.6 & & & \\
\hline
\end{tabular}

$\mathrm{MAC}=$ modal assurance criterion; $f_{e}=$ experimental frequency $\zeta=$ damping ratio; $\Delta f=$ frequency $(\mathrm{ERA})$ increase 
Table 3 Updating of the pre-upgrade bridge model

\begin{tabular}{|l|l|l|l|l|l|l|l|}
\hline \multirow{2}{*}{ Mode } & \multirow{2}{*}{$\mathrm{f}_{\mathrm{e}} / \mathrm{Hz}$} & \multicolumn{3}{|c|}{ Initial model } & \multicolumn{3}{|c|}{ Updated model } \\
\cline { 3 - 8 } & & $\left(f_{a}\right) / \mathrm{Hz}$ & $f_{a} / f_{e}$ & MAC & $\left(f_{a}\right) / \mathrm{Hz}$ & $f_{a} / f_{e}$ & MAC \\
\hline 1 & 5.55 & 5.23 & 0.944 & 0.887 & 5.84 & 1.053 & 0.884 \\
\hline 2 & 6.26 & 5.45 & 0.870 & 0.879 & 6.11 & 0.976 & 0.874 \\
\hline 3 & 7.53 & 6.28 & 0.834 & 0.921 & 7.22 & 0.958 & 0.918 \\
\hline 4 & 10.30 & 8.42 & 0.818 & 0.545 & 10.07 & 0.978 & 0.548 \\
\hline 5 & 14.76 & 12.30 & 0.833 & 0.947 & 15.18 & 1.028 & 0.946 \\
\hline- & - & 17.85 & - & - & 22.40 & - & - \\
\hline 6 & 20.81 & 20.92 & 1.005 & 0.177 & 23.34 & 1.122 & 0.190 \\
\hline 7 & 23.74 & 21.15 & 0.891 & 0.168 & 23.64 & 0.996 & 0.174 \\
\hline 8 & 30.74 & 21.77 & 0.708 & 0.351 & 24.45 & 0.795 & 0.354 \\
\hline
\end{tabular}

$f_{e}=$ experimental frequency; $f_{a}=$ analytical frequency; $\mathrm{MAC}=$ modal assurance criterion 
Table 4 Updating of the post-upgrade bridge model

\begin{tabular}{|l|l|l|l|l|l|l|l|}
\hline Mode & \multirow{2}{*}{$\mathrm{f}_{\mathrm{e}} / \mathrm{Hz}$} & \multicolumn{3}{|c|}{ Initial model } & \multicolumn{3}{l|}{ Updated model } \\
\cline { 3 - 8 } & & $\left(f_{a}\right) / \mathrm{Hz}$ & $f_{a} / f_{e}$ & MAC & $\left(f_{a}\right) / \mathrm{Hz}$ & $f_{a} / f_{e}$ & MAC \\
\hline 1 & 8.31 & 7.04 & 0.847 & 0.927 & 9.08 & 1.092 & 0.896 \\
\hline 2 & 9.34 & 7.18 & 0.769 & 0.983 & 9.16 & 0.981 & 0.981 \\
\hline 3 & 10.71 & 8.19 & 0.764 & 0.910 & 10.00 & 0.933 & 0.887 \\
\hline 4 & 12.95 & 10.88 & 0.840 & 0.974 & 12.31 & 0.951 & 0.976 \\
\hline 5 & 17.15 & 15.80 & 0.921 & 0.895 & 16.81 & 0.980 & 0.898 \\
\hline- & - & 22.74 & - & - & 23.44 & - & - \\
\hline 6 & 21.02 & 24.49 & 1.165 & 0.206 & 27.37 & 1.302 & 0.243 \\
\hline 7 & 26.89 & 24.57 & 0.914 & 0.060 & 27.41 & 1.019 & 0.062 \\
\hline 8 & 27.91 & 25.49 & 0.913 & 0.472 & 28.32 & 1.014 & 0.406 \\
\hline 9 & 32.92 & 27.34 & 0.831 & 0.234 & 29.99 & 0.911 & 0.222 \\
\hline
\end{tabular}

$f_{e}=$ experimental frequency; $f_{a}=$ analytical frequency; MAC $=$ modal assurance criterion 


\section{Figure Captions}

Figure 1a Pioneer Bridge before upgrading: elevation

Figure $1 \mathrm{~b} \quad$ Bridge soffit after upgrade showing inverted T-beams and snug fit on abutments.

Signal cables and piezo-electric accelerometers are visible.

Figure 1c Guard rail added during upgrading. Shaker and force-balance accelerometers are seen on the walkway

Figure 2 Detail of upgraded bridge at abutment showing rotational fixity due to rebars passing through deck and approach slabs

Figure 3 Acceleration time series due to passing vehicles

Figure 4 Pre-upgrade frequency response function (FRF) from accelerometer near shaker.

Units of FRF are inertance, $(1000 \mathrm{~kg})^{-1}$

Figure $5 \quad$ First five pre-upgrade modes from frequency domain eigensystem realisation algorithm ERA.

Figure $6 \quad$ Elevation (side) view of walkway components of three higher order pre-upgrade modes from frequency domain eigensystem realisation algorithm

Figure 7 Driving point frequency response function (FRF) from post-upgrade testing. Units of FRF are inertance, $(1000 \mathrm{~kg})^{-1}$

Figure 8 Post-upgrade modes from eigensystem realisation algorithm.

Figure 9 Elevation (side) view of walkway component of post-upgrade modes

Figure 10 Relative normalized sensitivities of responses to changes in model parameters for preupgrade bridge model

Figure 11 Pairing of experimental and analytical frequencies before and after updating for preupgrade bridge model

Figure 12 Relative normalized sensitivities of responses to changes in model parameters for postupgrade bridge model 
Figure 13 Pairing of experimental and analytical frequencies before and after updating for postupgrade bridge model

Figure 14 Elevation view of first mode shape along the bridge at the walkway showing partial fixity at the abutment. The solid line is the finite element model (FEM), circles represent experimental values. 


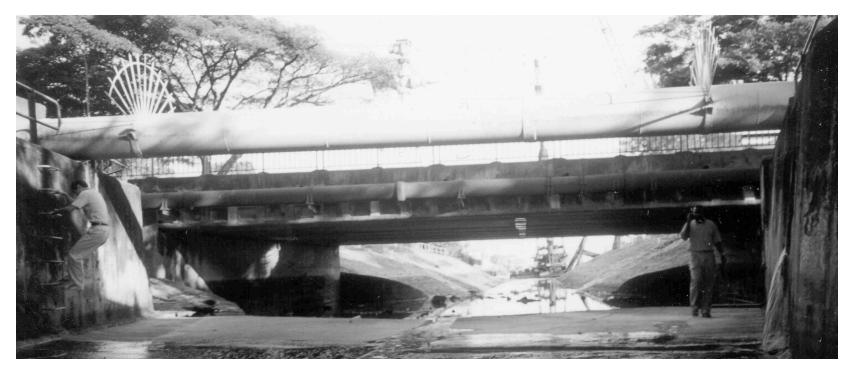

a)

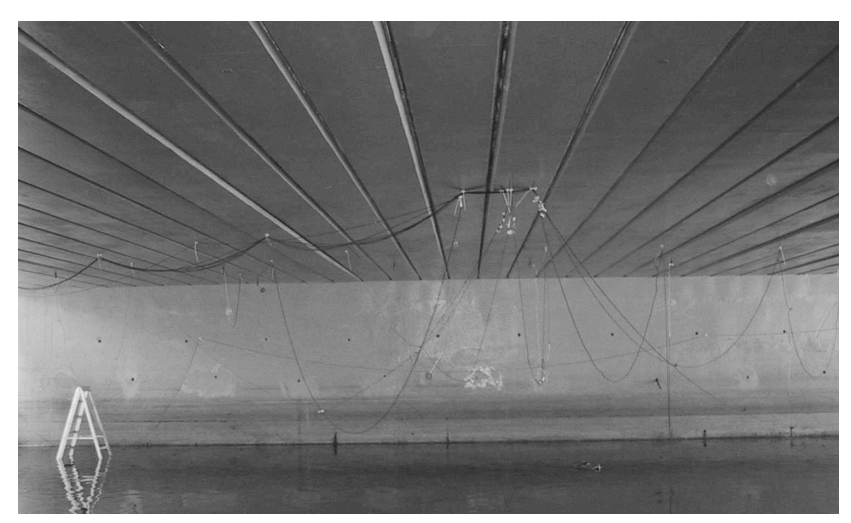

b)

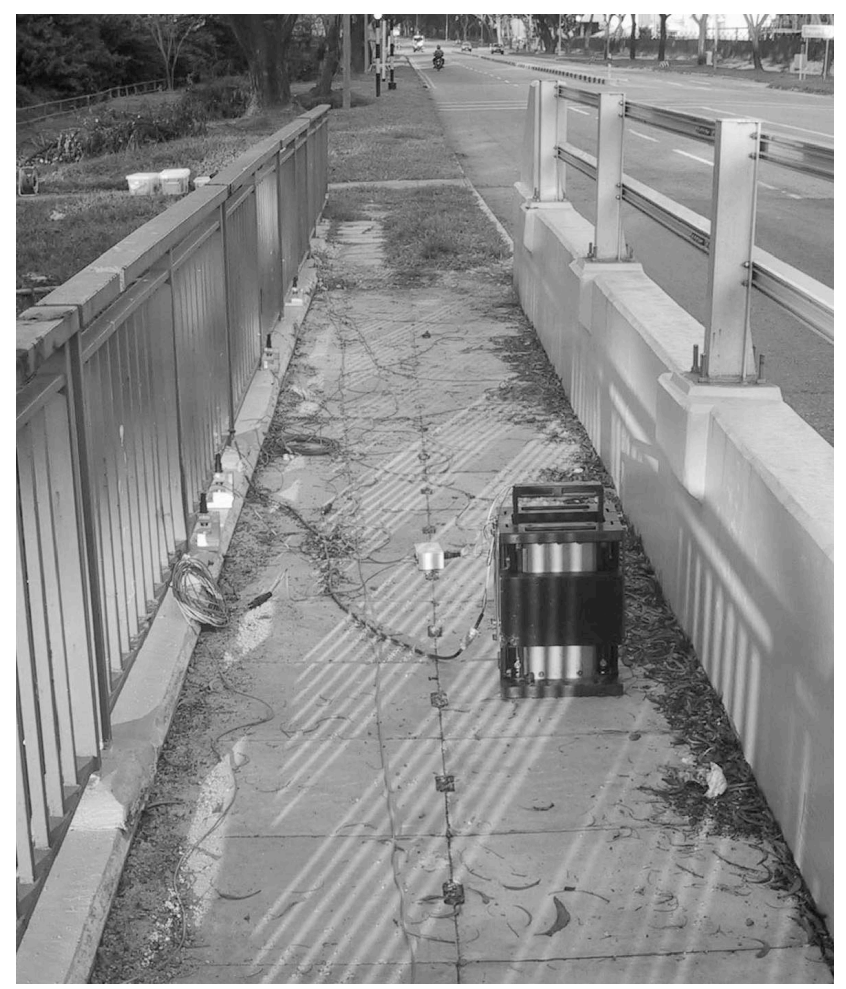

c)

FIG. 1 


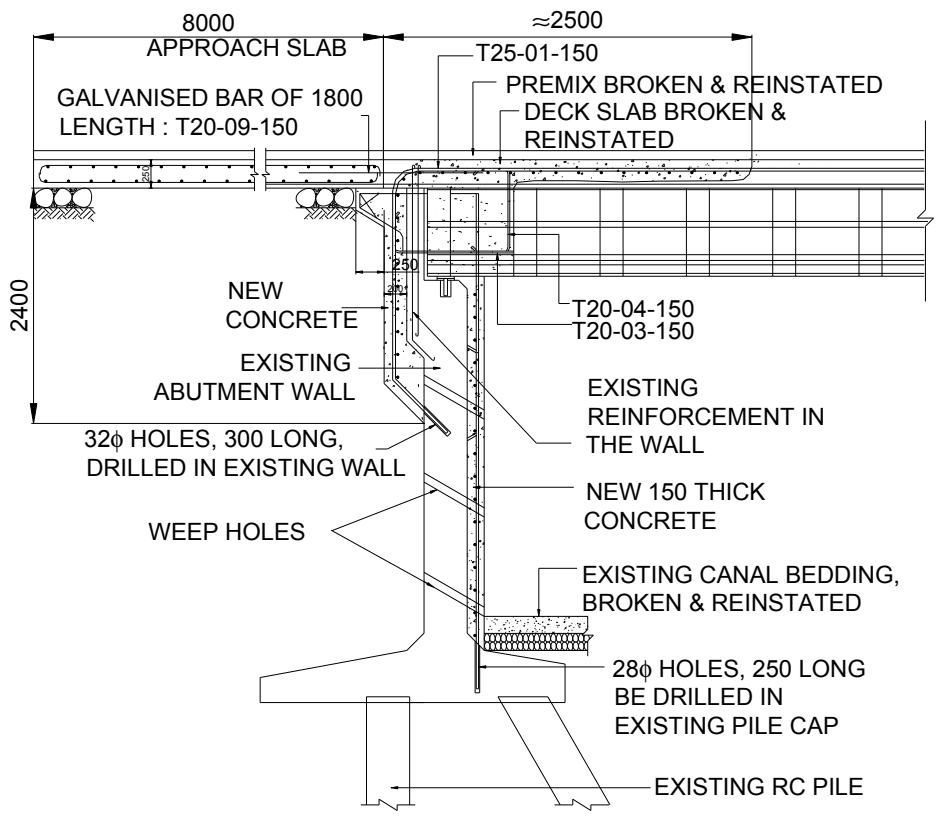

FIG. 2 


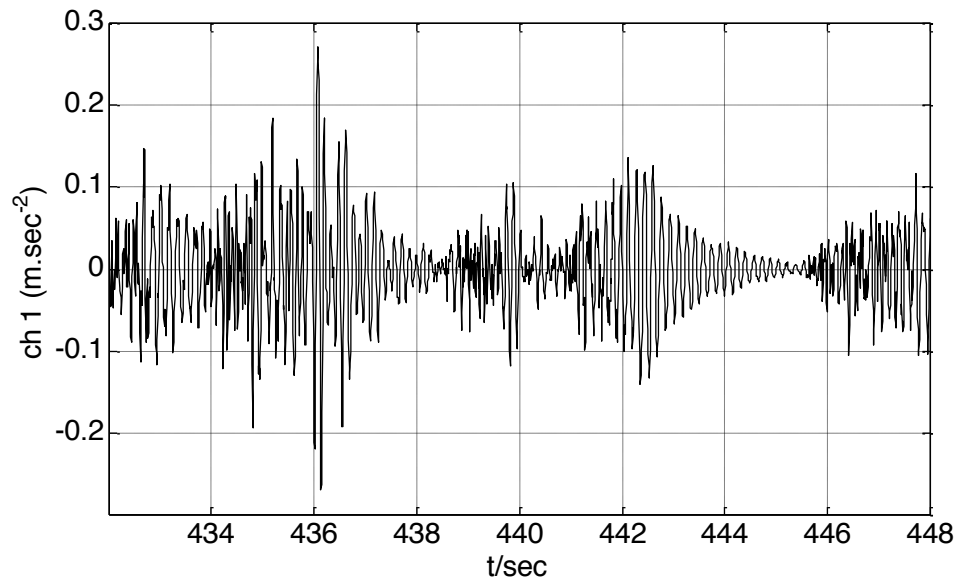

FIG. 3 

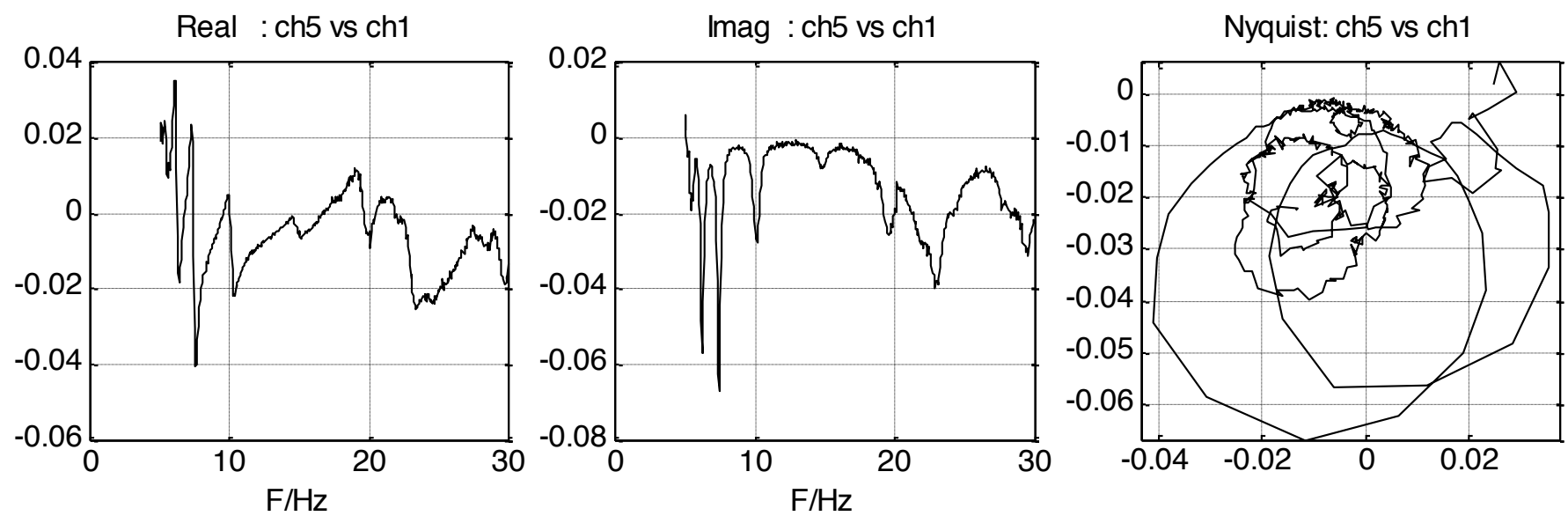

FIG. 4 


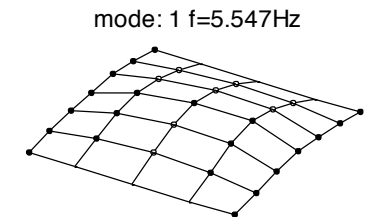

mode: $3 \mathrm{f}=7.534 \mathrm{~Hz}$

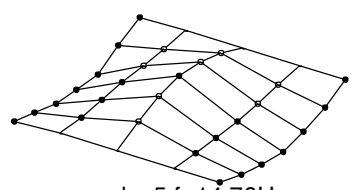

mode: $5 \mathrm{f}=14.76 \mathrm{~Hz}$

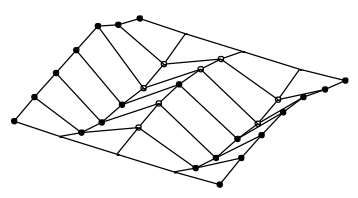

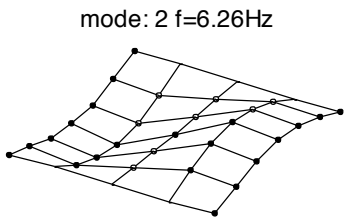

mode: $4 \mathrm{f}=10.3 \mathrm{~Hz}$

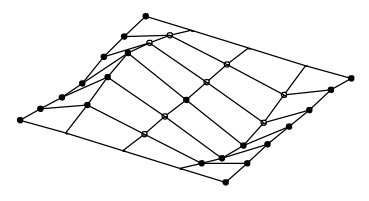

FIG. 5 


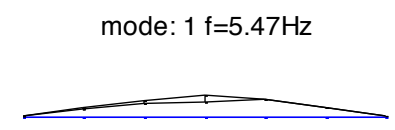

mode: $6 \mathrm{f}=20.81 \mathrm{~Hz}$

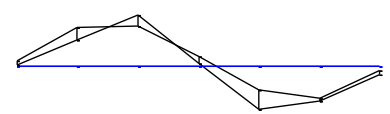

mode: $7 \mathrm{f}=23.74 \mathrm{~Hz}$

mode: $8 \mathrm{f}=30.74 \mathrm{~Hz}$
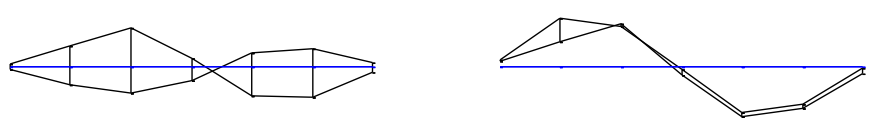

FIG. 6 

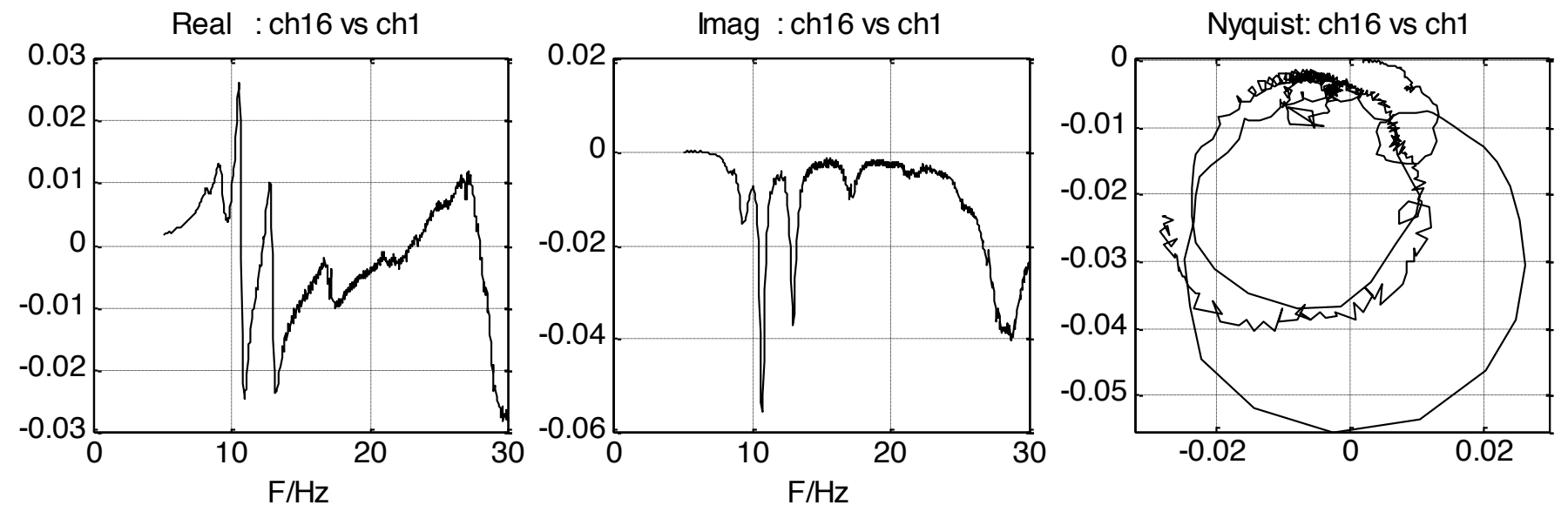

FIG. 7 

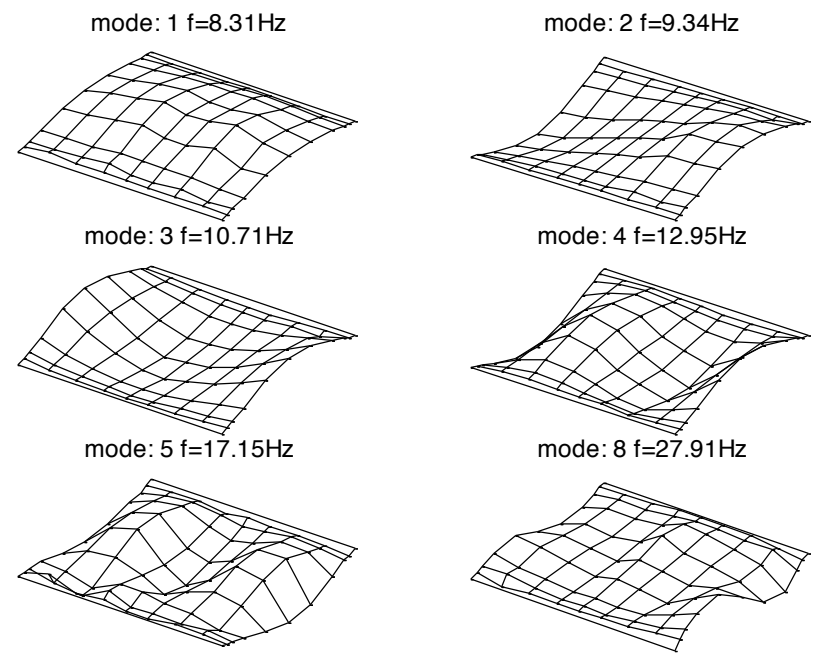

FIG. 8 


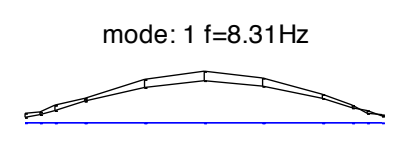

mode: $3 \mathrm{f}=10.71 \mathrm{~Hz}$

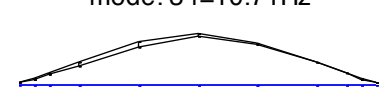

mode: $5 \mathrm{f}=17.15 \mathrm{~Hz}$

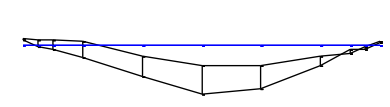

mode: $2 \mathrm{f}=9 \cdot 34 \mathrm{~Hz}$

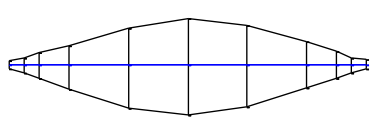

mode: $4 \mathrm{f}=12.95 \mathrm{~Hz}$

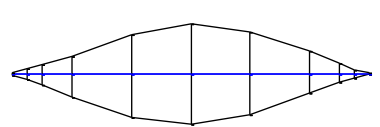

mode: $8 \mathrm{f}=27.91 \mathrm{~Hz}$

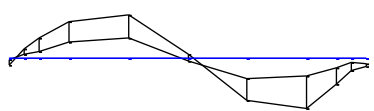

FIG. 9 


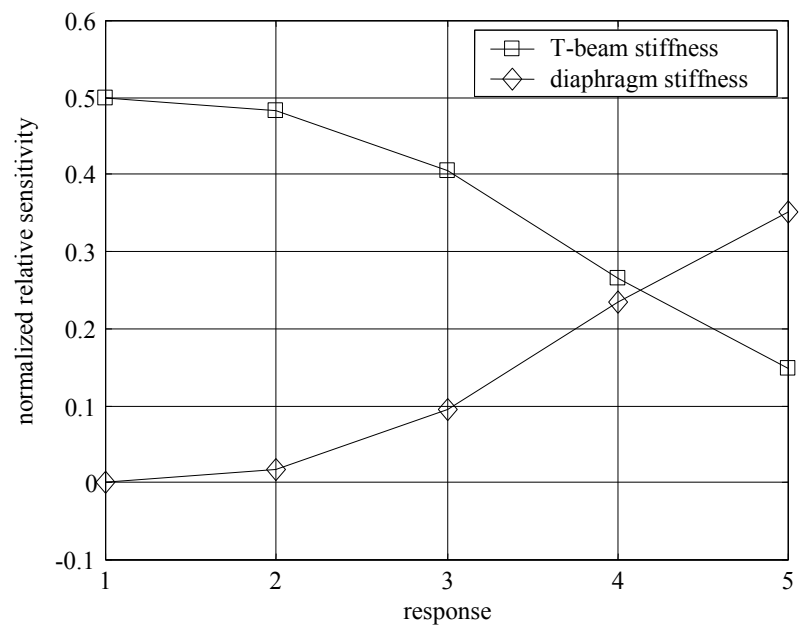

FIG. 10 


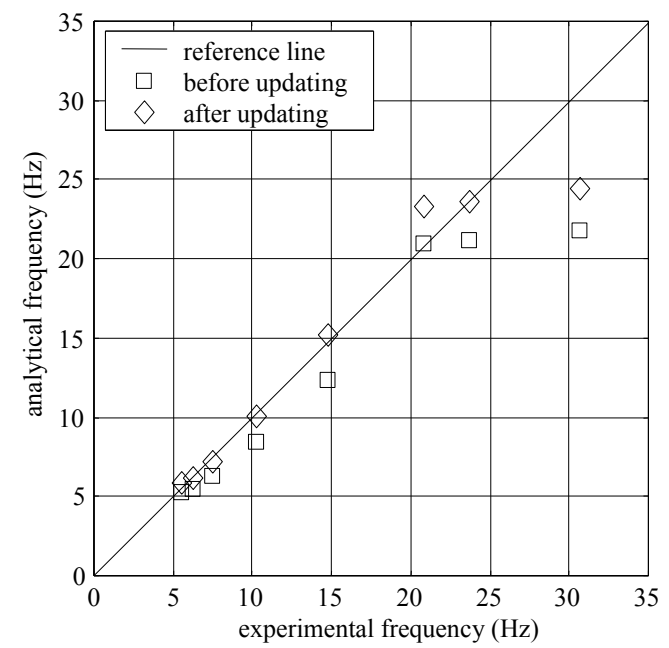

FIG. 11 


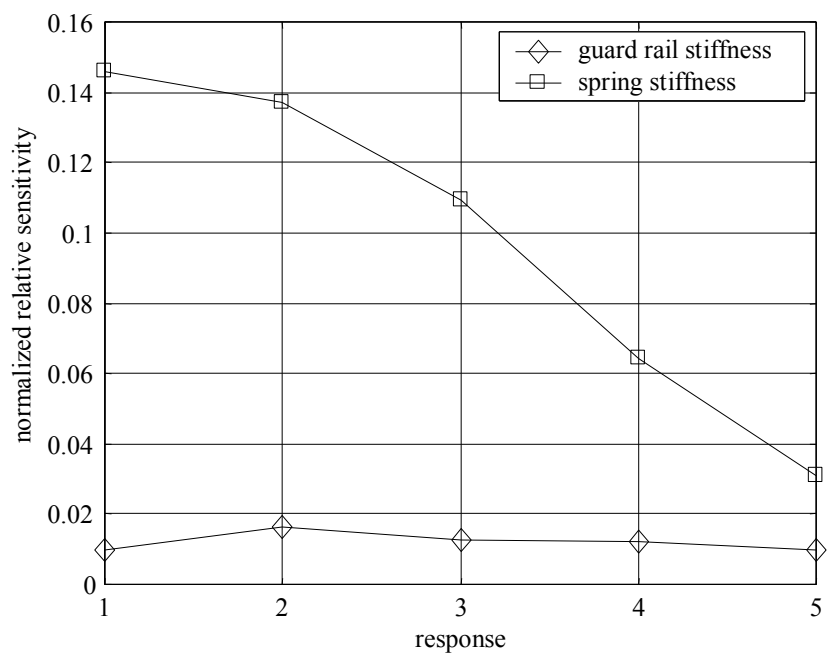

FIG. 12 


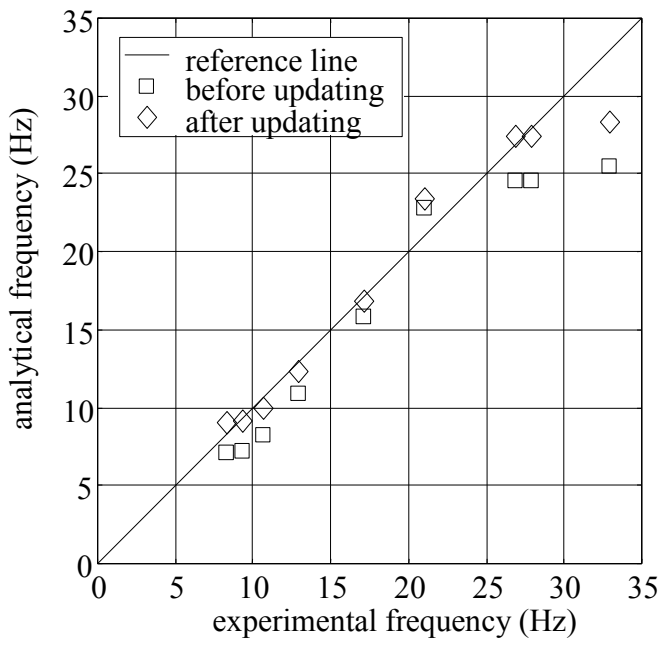

FIG. 13 


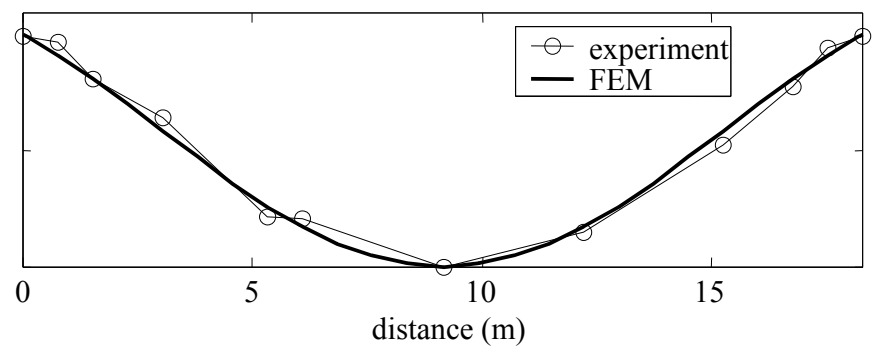

FIG. 14 